\title{
The clinical impact of observer variability in lung nodule classification in children with Wilms Tumour
}

\author{
Jesper Brok ${ }^{1}$, Susan Shelmerdine ${ }^{2}$, frederikke damsgaard ${ }^{3}$, Anne Smets ${ }^{4}$, Sabine IRTAN ${ }^{5}$, \\ Sophie Swinson ${ }^{6}$, Venus Hedayati ${ }^{7}$, Joseph $\mathrm{Jacob}^{8}$, Arjun Nair ${ }^{9}$, Minou Oostveen ${ }^{10}$, Kathy \\ Pritchard-Jones ${ }^{10}$, and Øystein Olsen ${ }^{2}$ \\ ${ }^{1}$ University College London Institute of Child Health \\ ${ }^{2}$ Great Ormond Street Hospital for Children \\ ${ }^{3}$ Copenhagen University Hospital \\ ${ }^{4}$ Academic Medical Centre \\ ${ }^{5}$ Hôpital Necker \\ ${ }^{6}$ Leeds Teaching Hospitals NHS Trust \\ ${ }^{7}$ King's College Hospital NHS Foundation Trust \\ ${ }^{8}$ University College London \\ ${ }^{9}$ University College London Hospitals NHS Foundation Trust \\ ${ }^{10}$ UCL Great Ormond Street Institute of Child Health Library
}

February 9, 2022

\begin{abstract}
Objectives To investigate the extent to which observer variability of CT lung nodule assessment may affect clinical treatment stratification in Wilms Tumor (WT) patients, according to the recent SIOP-RTSG UMBRELLA protocol. Methods I: CT thoraces of children with WT submitted for central review, were used to estimate size distribution of lung metastases. II: Scans were selected for blinded review by five radiologists to determine intra and inter-observer variability. They assessed identical scans on two occasions six months apart. III: Monte Carlo simulation (MCMC) was used to predict the clinical impact of observer variation when applying the UMBRELLA protocol size criteria. Results Lung nodules were found in 84 out of $360(23 \%)$ children with WT. For 21 identified lung nodules, inter-observer limits of agreement (LOA) for the five readers were $\pm 2.4 \mathrm{~mm}$ and $\pm 1.4 \mathrm{~mm}$ (AP diameter), $\pm 1.9 \mathrm{~mm}$ and $\pm 1.8 \mathrm{~mm}$ (TS diameter) and $\pm 2.0 \mathrm{~mm}$ and $\pm 2.4 \mathrm{~mm}$ (LS diameter) at assessments 1 and 2. Intra-observer LOA across the three dimensions were $\pm 1.5 \mathrm{~mm}, \pm 2.2 \mathrm{~mm}, \pm 3.5 \mathrm{~mm}, \pm 3.1 \mathrm{~mm}$ and $\pm 2.6 \mathrm{~mm}$ (readers 1-5). MCMC demonstrated that $17 \%$ of the patients with a 'true' nodule size of ?3mm will be scored as $<3 \mathrm{~mm}$, and $21 \%$ of the patients with a 'true' nodule size of $<3 \mathrm{~mm}$ will be scored as being ?3 mm. Conclusion A significant intra-inter observer-variation was found when measuring lung nodules on CT for patients with WT. This may have significant implications on treatment stratification, and thereby outcome, when applying a threshold of ?3 $\mathrm{mm}$ for a lung nodule to dictate metastatic status.
\end{abstract}

\section{Hosted file}

Lung nodule article submission Ped. blood and cancer 8:2.docx available at https://authorea. com/users/311377/articles/555899-the-clinical-impact-of-observer-variability-in-lungnodule-classification-in-children-with-wilms-tumour

\section{Hosted file}


Table 1.docx available at https://authorea.com/users/311377/articles/555899-the-clinicalimpact-of-observer-variability-in-lung-nodule-classification-in-children-with-wilmstumour

\section{Hosted file}

Table 2.docx available at https://authorea.com/users/311377/articles/555899-the-clinicalimpact-of-observer-variability-in-lung-nodule-classification-in-children-with-wilmstumour

\section{Hosted file}

Table 3.docx available at https://authorea.com/users/311377/articles/555899-the-clinicalimpact-of-observer-variability-in-lung-nodule-classification-in-children-with-wilmstumour

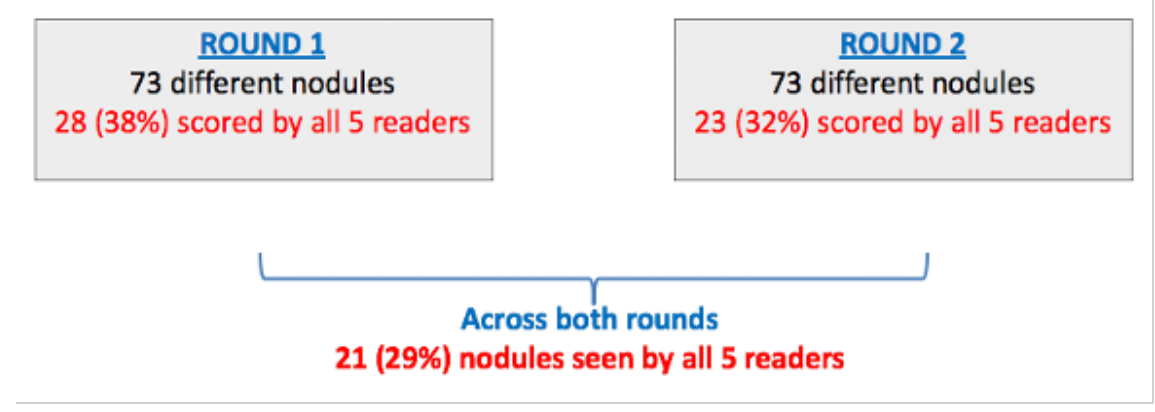

AP dimension $(n=21)$

Average $=6.1 \mathrm{~mm}$

Range $=1-21 \mathrm{~mm}$

LOA $= \pm 2.4 \mathrm{~mm}$

TS dimension ( $n=21$ )

Average $=5.7 \mathrm{~mm}$

Range $=1-17 \mathrm{~mm}$

LOA $= \pm 1.94 \mathrm{~mm}$

LS dimension $(\mathrm{n}=21)$

Average $=6.2 \mathrm{~mm}$

Range $=1-24 \mathrm{~mm}$

LOA $= \pm 2.02 \mathrm{~mm}$
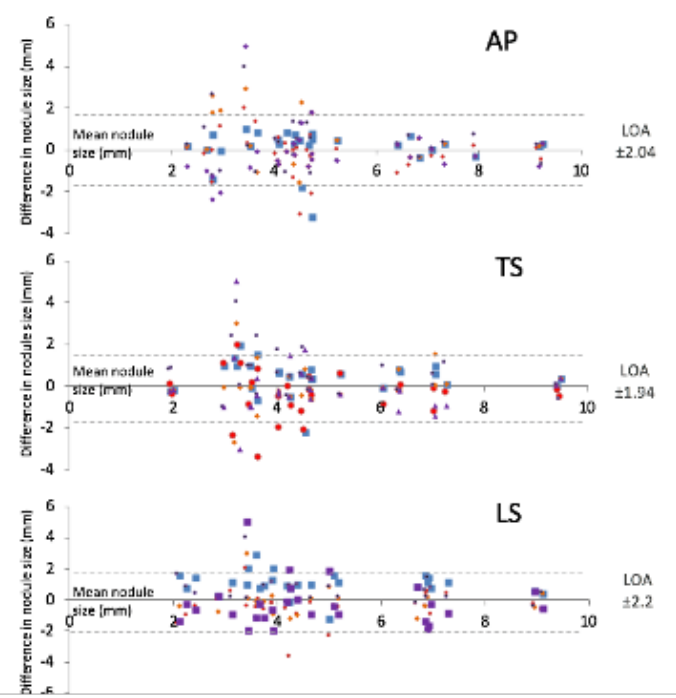


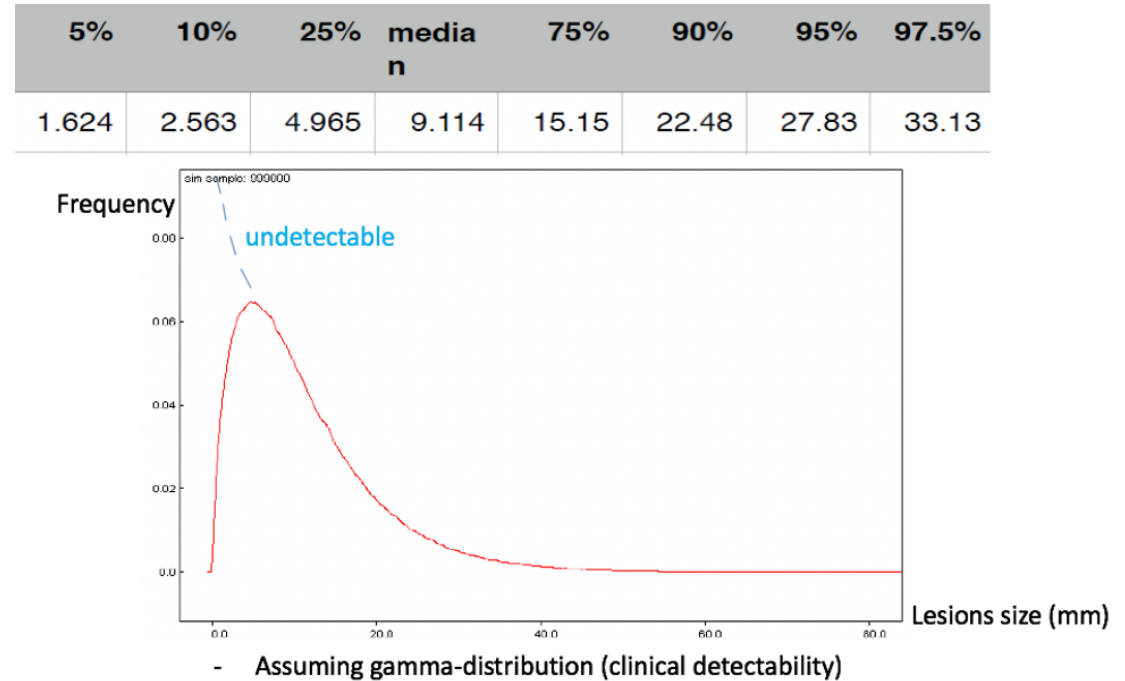

